\title{
ADAPTACIÓN, CRECIMIENTO Y SUPERVIVENCIA DE ALEVINES DE DONCELLA Pseudoplatystoma punctifer AL CONSUMO DE ALIMENTO BALANCEADO
}

\author{
Christian FERNÁNDEZ MÉNDEZ ${ }^{1,3}$, Diana CASTRO-RUIZ ${ }^{1,3}$, Carmen GARCÍA-DÁVILA ${ }^{1,3}$, Fabrice \\ DUPONCHELLE ${ }^{2,3}$, Jean François RENNO ${ }^{2,3}$, Jesús NÚÑ̃ZZ \\ 1 Instituto de Investigaciones de la Amazonía Peruana (IIAP). Carretera Iquitos Nauta km 4.5, Iquitos, Perú. Programa para \\ el Uso y Conservación del Agua y sus Recursos - AQUAREC, Apartado 784, Iquitos, Perú. E-mail: \\ cfernandez@iiap.org.pe \\ 2 Institut de Recherche pour le Développement (IRD), LMI EDIA, ISEM- UMR IRD 226, Place E. Bataillon, CC063, 34095 \\ Montpellier cedex 05, France. \\ 3 Laboratoire Mixte International - Evolution et Domestication de l'Ichtyofaune Amazonienne (LMI-EDIA)
}

\section{RESUMEN}

La doncella Pseudoplatystoma punctifer es uno de los grandes bagres de importancia comercial en la Amazonía, su carne es muy apreciada en los mercados locales debido a la ausencia de espinas intramusculares y al tamaño que alcanza. Se encuentra ampliamente distribuida en la cuenca amazónica, es una especie prometedora para la producción piscícola, pero en la actualidad las altas tasas de mortalidad en la etapa de alevinaje impiden un mayor desarrollo de su piscicultura. El presente estudio tuvo por finalidad adaptar al consumo de alimento balanceado a alevines de doncella, mediante el uso de una dieta intermedia húmeda (destete) en un sistema de recirculación de agua por un periodo de 130 días post-fertilización (dpf), con una frecuencia de alimentación inicial de 5 veces al día compuesta por nauplios de Artemia. La supervivencia se determinó mediante conteo directo. Se obtuvo como resultado una supervivencia de $32 \%$ hasta los $39 \mathrm{dpf}$ momento en que se inició el primer destete. La ausencia de mortalidad durante los 91 días restantes, el aumento en peso (promedio $7.76 \pm 2.46 \mathrm{~g}$ ) y longitud total $(106.74 \pm 10.97 \mathrm{~mm})$ alcanzados hasta los 130 dpf, demuestran el éxito de la adaptación de los alevines de esta especie al alimento seco.

PALABRAS CLAVE: Entrenamiento alimenticio, destete, levante larval.

\section{ADAPTATION, GROWTH AND SURVIVAL OF YOUNG FISH OF DONCELLA, Pseudoplatystoma punctifer TO THE CONSUMPTION OF BALANCED FOOD}

\begin{abstract}
"Doncella" Pseudoplatystoma punctifer is one of the biggest catfish in the Amazon with great commercial importance, its meat is highly prized in local markets due to the absence of intramuscular bones and to its size. It is widely distributed in the Amazon Basin, and represents a promising species for fish production, but until now facing high mortality rates in the fingerling stage obstructs further development of their farming. The present study aimed to adapt the consumption of balanced food to doncella's fingerlings, using an intermediate semi- humid diet (weaning) in a recirculating system for 130 days post fertilization (dpf), with an initial feed rate of 5 times a day composed for Artemia's naupli. The survival was estimated by direct count. The survival result was a $32 \%$ to $39 \mathrm{dpf}$ moment they initiated the first weaning. The absence of mortality during the 91 days remaining, the increase in weight (mean $7.76 \pm 2.46 \mathrm{~g})$ and total length $(106.74 \pm 10.97$ $\mathrm{mm}$ ) reached to $130 \mathrm{dpf}$, demonstrate the success of the adaptation of this species to fry food dry.
\end{abstract}

KEYWORDS: Food training, weaning, larval rearing. 


\section{INTRODUCCIÓN}

Una de las especies más conocidas de grandes bagres es la doncella Pseudoplatystoma punctifer, esta especie se encuentra ampliamente distribuida en la cuenca amazónica (Goulding et al., 1996). Es muy apreciada en los mercados locales de la Amazonia peruana, debido principalmente a la calidad de su carne, a la ausencia de espinas intramusculares y al tamaño que alcanza; esta especie también es considerada como una especie ornamental en las primeras fases de vida. Todo esto ha llevado al incremento de su demanda, que es cubierta por la extracción en el medio natural (Rodríguez, 1991).

Una de las alternativas para disminuir la presión de pesca sobre las poblaciones naturales, es el desarrollo de su crianza en cautiverio. En la actualidad existen tecnologías bien desarrolladas para su reproducción en sistemas controlados (Kossowski \& Madrid, 1985; Padilla et al., 2001; Gervasio et al., 2004; Nuñez et al., 2008). Sin embargo las altas tasas de mortalidad en la etapa larval y dificultades en la obtención de alevines (Kossowski \& Madrid, 1991, 1985; Padilla et al., 2001) dificulta actualmente el desarrollo de su piscicultura. Los primeros ensayos de levante de alevines, demostraron la existencia de un fuerte canibalismo en esta especie (Kossowski, 1991). La hibridación artificial de Pseudoplatystoma punctifer con otros bagres, en su mayoría de la familia Pimelodidae, fue evaluada como una posibilidad de obtener peces con características menos complicadas (menor tasa de canibalismo, mejor adaptación a dietas inertes, entre otros) sin mayores avances para su cultivo (Kossowski, 1991, 1992). A esto se suma la insuficiente tecnología para la producción de alevines "entrenados" al consumo de alimentos granulados o extruidos, que permitan la producción a gran escala de esta especie en América Latina (Kossowski, 1996; Nuñez, 2009). Sin embargo, recientemente se han realizado aportes significativos (protocolos de alimentación, densidad de siembra y destete) aplicados al cultivo en las primeras etapas post-larvales de la doncella (Gervasio et al., 2004; Nuñez et al., 2008; DíazOlarte et al., 2009). Existen ya protocolos de adaptación (destete) para el cultivo de alevinos de otra especie de bagre del mismo género (Pseudoplatystoma corruscans), lográndose protocolos para el "entrenamiento" a consumir dietas artificiales para la obtención de alevines (Martino et al., 2002; Guerrero, 2003; Segura et al., 2004). En tal sentido, los objetivos del presente estudio fueron adaptar al consumo de alimento balanceado a alevines de doncella Pseudoplatystoma punctifer mediante el uso de una dieta artificial intermedia húmeda, y evaluar el crecimiento y la supervivencia durante la fase de adaptación o entrenamiento.

\section{MATERIALES Y MÉTODOS}

El presente trabajo se realizó en el Centro de Investigaciones Fernando Alcántara Bocanegra (CIFAB) del Instituto de Investigaciones de la Amazonía Peruana (IIAP), el cual se encuentra ubicado a la margen derecha de la carretera Iquitos Nauta a $4.5 \mathrm{Km}$. de la ciudad de Iquitos, Distrito de San Juan Bautista, Región Loreto.

\section{OBTENCIÓN DE LARVAS POR INDUCCIÓN HORMONAL}

Las hembras seleccionadas para el proceso de inducción hormonal fueron aquellas que presentaron el abdomen más prominente o abultado y los machos que emitieron esperma por leve presión abdominal. La evaluación del estado de madures ovocitaria fue determinado por canulación. El tratamiento hormonal de los peces seleccionados fue realizado según el protocolo desarrollado por Padilla et al. (2001) para la especie.

La inducción se realizó mediante la inyección intramuscular de una hormona sintética (Conceptal ()), la primera dosis fue suministrada a las 18 horas del mismo día de la selección, la segunda a las 6 horas del día siguiente. Unas horas después de la segunda dosis hormonal, se realizó la colecta de esperma de los machos en jeringas que contenían suero fisiológico para evitar la activación del esperma. El semen diluido (1:5), fue homogenizado y mediante observación microscópica se verificó la inactivación del mismo durante el proceso de colecta. El desove se realizó después del mediodía ( 8 horas después de la segunda inyección). Inmediatamente después de la ovulación, los óvulos de la hembra fueron fertilizados con el esperma del macho seleccionado. Los óvulos fertilizados fueron hidratados con agua y acondicionados en las incubadoras de fibra de vidrio tipo Woynarovich, de 60 litros de capacidad con un flujo ascendente de agua de 0.3 L.s ${ }^{-1}$ a $27.5 \pm 5^{\circ} \mathrm{C}$.

\section{UNIDAD EXPERIMENTAL}

Se usó como unidad experimental un circuito cerrado de recirculación de agua, constituido por 6 unidades de 6 tanques de fibra de vidrio de 30 litros cada uno, con un flujo de agua constante $(0.5 \mathrm{~L}$. min $\left.{ }^{1}\right)$, cada tanque contenía una piedra difusora alimentada por un Blower para garantizar la oxigenación del sistema. Una resistencia con termostato permitía mantener una temperatura constante en cada módulo, y tres sistemas de filtración: mecánica, biológica y UV aseguraron la buena calidad de agua a lo largo del experimento.

\section{FASE EXPERIMENTAL}

La eclosión de las larvas en las incubadoras se efectuó a 1 día post fertilización (dpf). A continuación se realizó la limpieza, primer conteo y 
traslado de las larvas a los tanques de concreto revestidos con mayólica, con flujo abierto de agua de 1 L.min ${ }^{-1}$. A los 5 dpf se efectuó el segundo conteo de las larvas y fotografía de un mínimo de 15 individuos para determinar su longitud total. Inmediatamente se trasladaron 1500 individuos a cada tanque de $30 \mathrm{~L}$ de la unidad experimental (circuito cerrado). Los 36 tanques fueron cubiertos con mantas plásticas negras para buscar el mejor desarrollo sin interacciones del entorno durante todo el experimento. Todos los tanques fueron sometidos a condiciones homogéneas de agua, temperatura y alimentación.

\section{OBTECION DE NAUPLIOS DE ARTEMIA (ALIMENTO INICIAL)}

La alimentación inicial de las larvas de doncella Pseudoplatystoma punctifer fue a base de nauplios de Artemia sp. recién eclosionados. Para lo cual se hidrato los cistos por espacio de una hora con abundante aireación, inmediatamente después los cistos fueron incubados en baldes translúcidos de 20 litros con agua a $25 \%$ de sal y temperatura de $28{ }^{\circ} \mathrm{C}$ durante 24 horas. Los cistos fueron mantenidos en baldes con iluminación y aireación artificial constante hasta que culminaron la eclosión de los nauplios a las 24 horas de incubación. Antes de la alimentación de las larvas de doncella se realizaron tres conteos de nauplios para determinar su densidad y determinar el volumen a suministrar en los tanques.

\section{ELABORACIÓN DE LA DIETAS}

La dieta artificial húmeda denominada "flan balanceado" posee 2 insumos: el alimento balanceado molido (alimento extruido para truchas de $6 \mathrm{~mm}$ al $40 \%$ de proteína) y colapez a una proporción de $96 \%$ y $4 \%$ respectivamente. Para la preparación del flan, al alimento balanceado se le adiciono agua a $40{ }^{\circ} \mathrm{C}$ hasta generar una pasta uniforme y finalmente se adicionó el colapez (previamente disuelto en agua recién hervida). Esta pasta fue bien homogenizada, inmediatamente moldeada y refrigerada $\left(4{ }^{\circ} \mathrm{C}\right)$ en recipientes de plástico dentro de bolsas "ziploc".

\section{ALIMENTACIÓN DE ALEVINOS DE DONCELLA}

En términos generales las larvas de doncella fueron alimentadas de los 3 a los 38 dpf exclusivamente con nauplios de artemia. A partir de los $39 \mathrm{dpf}$ se redujo gradualmente los nauplios y se incrementó la administración de una dieta inerte húmeda (proceso de adaptación). A partir de los $15 \mathrm{dpf}$ se redujo gradualmente la densidad de las larvas, numero de alimentaciones y porcentaje de alimento distribuido tal como se muestra en la Tabla 1.

\section{Alimentación con nauplios}

La primera alimentación se efectuó a los 3 dpf con $20 \%$ de la biomasa en alimento (nauplios) distribuido al día. Del 4 al 14 dpf se suministró el alimento a una frecuencia de 5 veces al día $(6,10,14$, 18 y 22 horas). La cantidad de nauplios suministrados por larva fue incrementada cada día proporcionalmente según el cuadro de crecimiento y controles puntuales. Se consideró como tiempo de alimentación una hora, siendo el flujo de agua detenido durante este período de tiempo, para facilitar la ingesta y evitar la salida de los nauplios por los filtros. Concluida la hora se realizaba la limpieza de los tanques mediante sifoneo, retirando el alimento no consumido, otros desperdicios y reponiendo el flujo de agua.

\section{Proceso de adaptación}

A partir del 39 dpf se inició con el primer período de adaptación a la primera dieta artificial húmeda denominada "flan balanceado". La dieta era retirada del molde y cortada en pequeñas porciones aproximadas para cada tanque según el porcentaje de suministro diario (10\%) mostrado en la Tabla 1. Las pequeñas porciones se pasaban por un tamiz de 1.5 $\mathrm{mm}$ para formar pequeños pellets húmedos alargados que iban al fondo del tanque y eran fáciles de ingerir por los alevines. Durante 8 días el suministro de la dieta inerte fue incrementándose progresivamente y reduciéndose gradualmente la administración de nauplios hasta llegar al 100\% de la dieta artificial "flan balanceado" y $0 \%$ de nauplios. Se ha conservado el período de tiempo de una hora para la alimentación con el flujo de agua detenido y quitando la aireación para mantener la consistencia del alimento en el agua. Cumplida la hora de suministrada la dieta se realizó la limpieza del alimento no consumido y finalmente se reinició el flujo de agua y la aireación. El suministro de esta dieta artificial "flan balanceado" continuó durante 70 días, reduciéndose la frecuencia de alimentación a 4 veces al día, cada 5 horas $(6,11,16$, y 21 horas). La administración durante este período se realizó con un tamiz de $2 \mathrm{~mm}$ el cuál se usó hasta los $70 \mathrm{dpf}$. A partir de los $71 \mathrm{dpf}$ y hasta los $90 \mathrm{dpf}$ se usó un tamiz de $3 \mathrm{~mm}$ para la administración de la dieta. Finalmente desde los $91 \mathrm{dpf}$ hasta el final del segundo proceso de adaptación se usó un tamiz de 5 $\mathrm{mm}$.

El segundo proceso de adaptación se inició a los 109 dpf y se realizó con los alevines distribuidos en 12 tanques de concreto rectangulares recubiertos de mayólicas (densidad de 1 individuo por litro), con flujo constante de agua, cubiertos con mantas plásticas negras y frecuencia de alimentación de 3 veces al día (6, 14, y 22 horas). En este proceso de manera similar al anterior proceso se redujo de manera gradual la administración de la dieta "flan 
balanceado", incrementándose simultáneamente el suministro del "alimento balanceado seco" (alimento extruido para truchas de $6 \mathrm{~mm}$ al $40 \%$ de proteína) hasta llegar al $100 \%$ de suministro del alimento balanceado y $0 \%$ de la dieta semi-húmeda "flan balanceado". Culminado este proceso de adaptación, la frecuencia de alimentación fue reducida a 2 veces al día ( 6 y 18 horas). Finalmente se mantuvo la alimentación con "alimento balanceado" durante 14 días (130 dpf) para constatar que los peces se encontraban adaptados al alimento balanceado.

\section{ANÁLISIS DE DATOS}

El porcentaje de supervivencia fue analizada a partir del conteo directo de los alevines. La longitud total de los peces fue analizada a partir de fotografías con ayuda del software libre de procesamiento de imágenes "Image J" y los datos se analizaron usando ANOVA con el software estadístico "Statgraphics Plus".

\section{RESULTADOS Y DISCUSIÓN}

La eclosión se realizó entre las 15 y 18 horas de incubación a $27 \pm 0.5^{\circ} \mathrm{C}$, lo cual representa un valor bastante similar a otros experimentos que reportan $14 \pm 2 \mathrm{hpf}$ a $27^{\circ} \mathrm{C}$ (Padilla et al., 2001) y $17.6 \mathrm{hpf}$ a $25{ }^{\circ} \mathrm{C}$ (Kossowski \& Madrid, 1985) para la misma especie. El porcentaje de eclosión (figura 1) de las larvas fue de $95.6 \%$ (89.7\% de larvas normales y $5.9 \%$ de deformes), este resultado se debe probablemente a la buena selección de los reproductores y al control de la calidad de agua del sistema de incubación. El porcentaje obtenido en este estudio esta sobre el rango de eclosión reportados para 23 eventos reproductivos $(40.76 \%$ a $92.96 \%$ ) evaluados por Nuñez et al. (2008).

Las longitudes totales $(\mathrm{mm})$ obtenidas durante los 6 controles $(\mathrm{C} 1-\mathrm{C} 6)$ y los coeficientes de variación $(\mathrm{CV} \%)$ durante los días de post-fertilización (dpf) están resumidos en la Tabla 2. El experimento se inició a los $5 \mathrm{dpf}$ con una longitud total promedio de $5.85 \mathrm{~mm}$ y culminó a los $130 \mathrm{dpf}$ con $106.74 \mathrm{~mm}$, no se encontró diferencia significativa entre las réplicas $(\mathrm{p}>0.05)$. El coeficiente de variación se incrementó de 6.69 a 9.73, mostrando un incremento de la heterogeneidad a medida que las post larvas incrementaron de tamaño. El promedio de longitud total obtenido a los $5 \mathrm{dpf}$ es similar a la reportada por Padilla et al., 2001 (alrededor de $5.82 \mathrm{~mm}$ ). Para los $15 \mathrm{dpf}$ las longitudes totales son bastantes similares $(12.00 \mathrm{~mm})$ a las obtenidas por Montalván (2008) a los $16 \mathrm{dpf}$ usando el mismo sistema de recirculación y alimentación con nauplios. La diferencia de longitud podría ser debido a las diferentes frecuencias de alimentación con nauplios de
Artemia y a características propias de cada puesta (Nuñez et al., 2008). El coeficiente de variación en la longitud total se incrementó desde la alimentación con nauplios $(6.69 \%)$ hasta el consumo completo de alimento balanceado (9.73\%), lo que puede ser explicado debido a que los nauplios por su distribución homogénea en el agua brindan la misma posibilidad de alimentarse a todos los peces y presentan una mejor aceptación en comparación con el alimento inerte, donde algunos tienen más posibilidad o cercanía para consumirlo, induciendo un menor gasto de energía y obteniendo la ventaja de ganar tamaño más rápidamente (Nuñez et al., 2008). El peso promedio final de los alevines a los $130 \mathrm{dpf}$ fue de $7.76 \pm 2.46 \mathrm{~g}$.

La supervivencia durante el experimento (Figura 2) fue de $44 \%$ entre $1-5 \mathrm{dpf}$, reduciéndose a $33 \%$ durante los 10 siguientes días del experimento. La causas de la mortalidad observada durante los primeros 5 dpf pueden ser mejor explicadas por las heridas generadas por mordeduras entre los individuos que por canibalismo propiamente dicho (Montalvan, 2008). Mientras que la mortalidad (33\%) para el periodo entre los 5 y 15 dpf corresponde a la etapa de mayor riesgo de canibalismo $(7-11 \mathrm{~mm})$ cuando el tamaño de la boca de los individuos más grandes es mayor a la altura del cuerpo de las larvas más pequeñas, lo que facilita el canibalismo (Baras, 2005; Montalvan, 2008). La conducta caníbal es inevitable en las especies carnívoras durante sus primeros estadios de vida (Qin \& Fast, 1996), pero puede ser controlado en cierta media al aumentar la frecuencia de alimentación y al mantener a las post larvas en la oscuridad (Nuñez et al., 2008). Para los 15 dpf otros estudios de post larvas doncella criadas en agua verde consumiendo zooplancton natural muestran que no hay mejor desempeño de la supervivencia (Nuñez et al., 2008). Mientras que una alimentación con nauplios a $15 \mathrm{dpf}$ permite una supervivencia de 54 a $72 \%$ (Montalvan, 2008). A los 17 dpf Marciales-Caro et al. (2010) muestran mejores resultados en la supervivencia de las post-larvas de doncella alimentadas con cladóceros $(58 \%)$ frente a la Artemia (42\%). A partir del 15 dpf hasta $39 \mathrm{dpf}$ se mantuvo bastante alta $(98 \%$ de los peces distribuidos a $15 \mathrm{dpf}$ ) frente a otros trabajos donde la supervivencia es muy variable, posiblemente debido a la aceptación parcial de las dietas inertes y a la ausencia de dietas húmedas intermedias (Machado, 1998; Guerrero, 2003). La supervivencia se mantuvo al $100 \%$ desde el inicio del proceso de adaptación al alimento inerte (39 dpf) hasta la culminación del experimento (130 dpf), debido a la aceptación total del alimento balanceado y la baja densidad que se mantuvo en este trabajo durante el proceso de adaptación. 


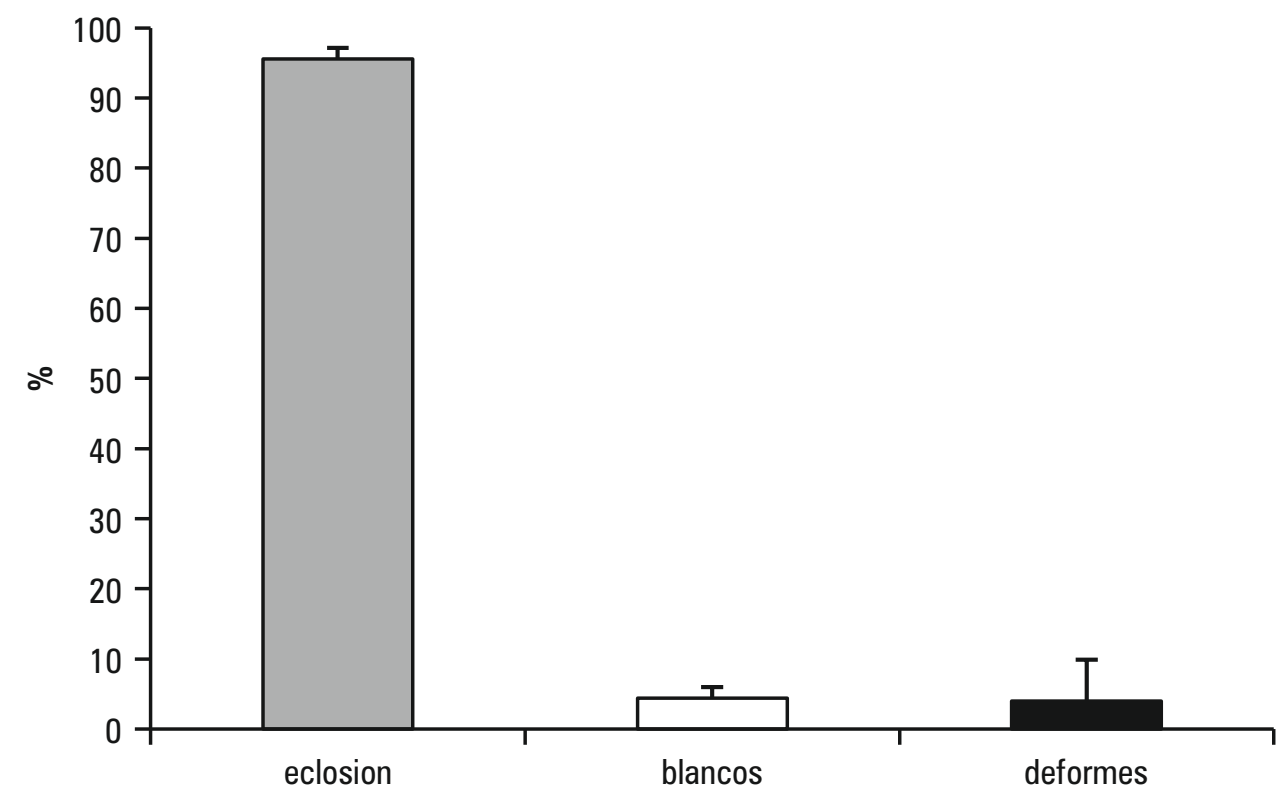

Figura 1. Promedios de los porcentajes de eclosión de larvas, blancos y deformes. Las líneas verticales sobre las barras representan la desviación estándar.

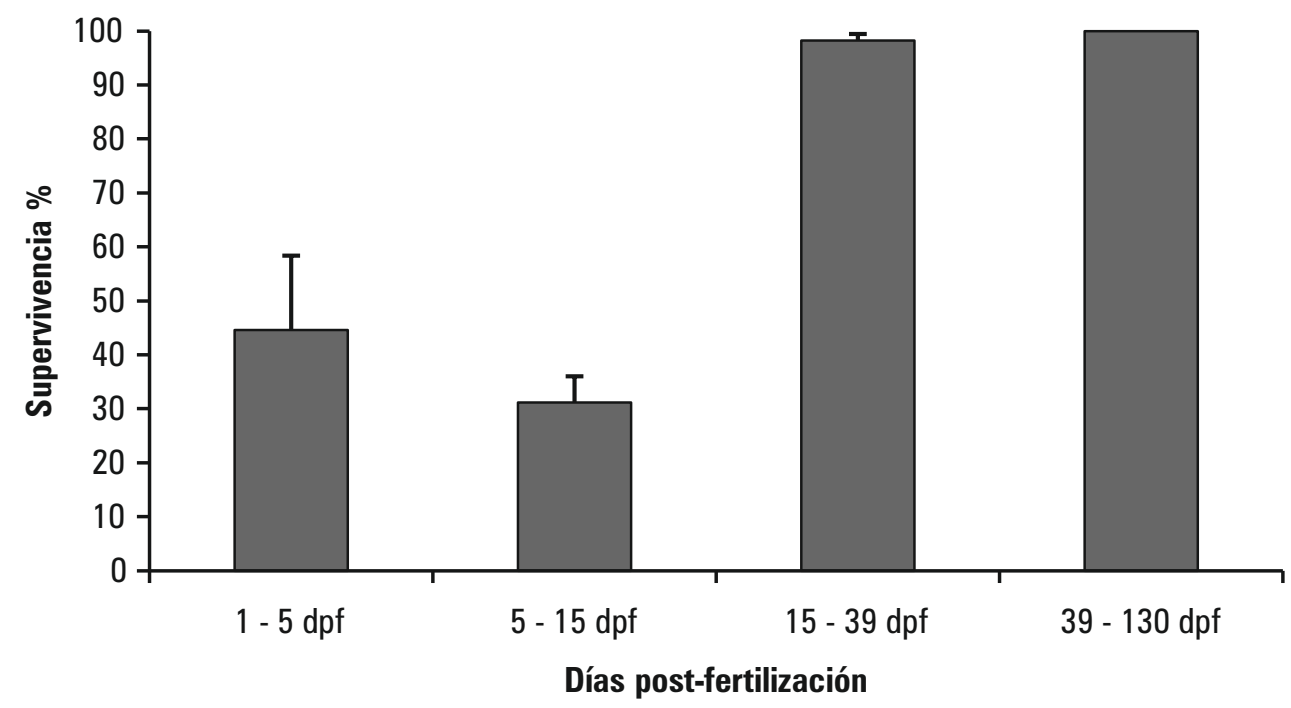

Figura 2. Promedios de los porcentajes de supervivencia sucesivos durante las cuatro fases de levante larval (130 días post-fertilización). Las líneas verticales sobre las barras representan la desviación estándar. 
Tabla 1. Densidad y número de alimentaciones al día en días post fertilización (dpf)

\begin{tabular}{lcccc}
\hline & \multicolumn{4}{c}{ Días post-fertilización (dpf) } \\
\cline { 2 - 5 } & $\mathbf{5 - 1 4}$ & $\mathbf{1 5 - 3 8}$ & $\mathbf{3 9 - 9 0}$ & $\mathbf{9 1 - 1 3 0}$ \\
\hline Densidad (ind. $\mathrm{L}^{-1}$ ) & 50 & 10 & 2 & 1 \\
Número de alimentaciones al día & 5 & 5 & 4 & 3 \\
\% de alimento distribuido/día & 20 & 15 & 10 & 5 \\
\hline
\end{tabular}

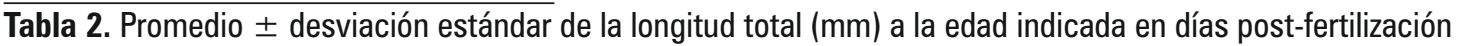
(dpf) y el coeficiente de variación (CV) correspondiente.

\begin{tabular}{|c|c|c|c|c|c|c|}
\hline & \multicolumn{6}{|c|}{ Controles } \\
\hline & $\begin{array}{c}\text { C1 } \\
5 \mathrm{dpf})\end{array}$ & $\begin{array}{c}\text { C2 } \\
\text { (10 dpf) }\end{array}$ & $\begin{array}{c}\text { C3 } \\
\text { (15 dpf) }\end{array}$ & $\begin{array}{c}\text { C4 } \\
\text { (52 dpf) }\end{array}$ & $\begin{array}{c}\text { C5 } \\
\text { (105 dpf) }\end{array}$ & $\begin{array}{c}\text { C6 } \\
\text { (130 dpf) }\end{array}$ \\
\hline$(\mathrm{mm})$ & $5.85 \pm 0.39$ & $8.37 \pm 0.75$ & $11.53 \pm 1.47$ & $46.46 \pm 9.04$ & $93.70 \pm 10.53$ & $106.74 \pm 10.97$ \\
\hline CV\% & 6.69 & 8.41 & 8.47 & 8.31 & 9.90 & 9.73 \\
\hline
\end{tabular}

\section{CONCLUSIONES}

Los resultados muestran que es posible adaptar alevines de doncella al consumo de alimento balanceado mediante el uso de una dieta húmeda intermedia. Asimismo, la dieta intermedia húmeda brinda la facilidad de realizar un proceso de adaptación gradual, que se ve reflejado en la supervivencia de $100 \%$ desde el primer proceso de adaptación hasta el final del experimento. Además, el uso de alimentos inertes permite un mejor control de la alimentación y mantiene la homogeneidad entre individuos, reduciendo el canibalismo y la dominancia de individuos de mayor tamaño.

\section{AGRADECIMIENTOS}

Al Proyecto Innovación y Competitividad para el Agro Peruano - INCAGRO, por el financiamiento parcial del presente estudio a través del subproyecto "Mejoramiento genético y producción intensiva de alevinos seleccionados de doncella Pseudoplatystoma fasciatum (Linnaeus, 1776) en la Amazonía peruana“.

\section{BIBLIOGRAFÍA CITADA}

Baras, E. 2005. Minimización del canibalismo en especies de peces con larvas piscívoras: estrategias y éxitos con el carácido Brycon moorei. Biología de las Poblaciones de Peces de la Amazonía y Piscicultura. Memorias del Primer Coloquio Internacional de la Red de Investigación sobre la Ictiofauna Amazónica, Iquitos, Perú. p. 227-233.

Díaz-Olarte, J.; Cruz, N.; Marciales, L.; Medina, V.; Cruz, P. 2009. Efectos de la densidad de siembra y disponibilidad de alimento sobre el desarrollo y sobrevivencia de larvas de Pseudoplatystoma fasciatum. Orinoquia, 13(1):21-30.

Gervásio, L.; Romagosa, E.; Borella, M.; Batlouni, S. 2004. Induced spawning of hatchery-raised Brazilian catfish, cachara Pseudoplatystoma fasciatum (Linnaeus, 1766). Aquaculture, 240: 451-461.

Guerrero, C. E. 2003. Treinamento alimentar de pintado Pseudoplatystoma coruscans (Agassiz. 1829): Sobrevivencia, crescimento e aspectos 
económicos. Tesis de Maestría, Centro de Aquicultura Jaboticabal; Universidade Estadual Paulista, São Paulo, Brasil. Unillanos, Villavicencio, Meta. 65-67 pp.

Goulding, M.; Smith, N.J.H.; Mahar. D.J. 1996. Floods of fortune. Ecology and Economy along the Amazon. Columbia University Press.

Kosswski, C.; Madrid, F. 1985. Ensayo de la reproducción inducida en el bagre rayado cabezón Pseudoplatystoma fasciatum (Linnaeus 1766) (Pisces. Siluriformes). Acta Científica Venezolana, 36: 284-285.

Kossowski, C. 1991. Experiencias iniciales sobre la hibridizacion de Leiarius marmoratus (Gill) 1871 por Pseudoplatystoma fasciatum (Linnaeus 1766) (Pisces, Siluriformes). Acta Cientifica Venezolana, 42: 48-50.

Kossowski, C.; Madrid, F. 1991. Observaciones de los Estadios Embrionario y Larval del bagre rayado cabezón Pseudoplatystoma fasciatum (Linnaeus 1766) (Pisces, Siluriformes). Biollania, 8: 9-15.

Kossowski, C. 1992. Avances en la hibridación artificial de Pseudoplatystoma fasciatum (Linnaeus) 1766 por Leiarius marmoratus (Gill) 1766 y (Pises, Siluriformes, Pimelodidae). Boletín Red de Acuicultura, 6 (1): 3-7

Kossowski, C. 1996. Perspective de L'élevage dês poisons-chats (Siluroidei) en Amérique du Sud. Aquat. Living Resour, Hors Série: 189-195.

Machado, J.H.; Del-Carrote, C.R.; Garossino, A.P.R.; Mazeto, M.D.; Grechi, F.C.S. 1998. Treinamento alimentar para aceitaçao de raçoes artificiais em alevinos de pintado (Pseudoplatystoma coruscans). Simpósio brasileiro de aquicultura, 10: 101-108.

Marciales-Caro L.; Díaz-Olarte J.; Medina-Robles V.; Cruz-Casallas P. 2010 Evaluación del crecimiento y sobrevivencia de larvas de bagre rayado Pseudoplatystoma fasciatum (Linneaus, 1766) alimentadas con alimento vivo natural y enriquecido con ácidos grasos. Revista Colombiana de Ciencia Pecuaria, 23:308-316.

Martino, R.C.; Cyrino, J.E.; Portz, L.; Trugo, L.C. 2002. Effect of dietary lipid level on nutritional performance of the surubim, Pseudoplatystoma coruscans. Aquaculture, 209: 209-218.

Montalván, G.V. 2008. Ontogenia de la doncella, Pseudoplatystoma fasciatum (Linnaeus, 1766), $\mathrm{y}$ su relación con la dinámica del canibalismo. Tesis para optar el Título Profesional de Ingeniero Pesquero Acuicultor. Lima: Universidad Nacional Federico Villarreal. Lima. Perú. 68 pp.

Nuñez, J.; Dugué, R.; Corcuy, N.; Duponchelle, F.; Renno, J. F.; Raynaud, T.; Hubert, N.; Legendre, M. 2008. Induced breeding and larval rearing of Surubi, Pseudoplatystoma fasciatum (Linnaeus, 1766), from the Bolivian Amazon. Aquaculture Research, 39: 764-776.

Nuñez, J. 2009. Domesticación de nouvelles espèces d'intérêt piscicole en Amazonie. Cah Agric, vol. 18(2-3).

Padilla, P.; Alcántara, F.; Ismiño, R. 2001. Reproducción inducida de la doncella Pseudoplatystoma fasciatum y desarrollo embrionario - larval. Folia Amazónica 12(12):141-154.

Qin, J.; Fast, A.W. 1996. Size and feed dependent cannibalism with juvenile snakehead Channa striatus. Aquaculture, 144: 313-320.

Rodríguez, C.A. 1991. Bagres malleros y cuerderos en el bajo río caqueta (Amazonía colombiana). Comercial fisheries in the lower caquetá river. Estudios de la Amazonía Colombiana. Vol. 2 programa Tropenbos Colombia. $280 \mathrm{pp}$.

Segura, L.; Hayashi C.; De Souza S.; Soares C. 2004. Canibalismo entre larvas de pintado, Pseudoplatystoma corruscans, cultivadas sobre diferentes densidades de estocagem. Acta Scientiarum Biological Sciences, 26(3) 299-302. 
